\title{
The Modified Sadik Decomposition Method to Solve a System of Nonlinear Fractional Volterra Integro-Differential Equations of Convolution type
}

\author{
PRAPART PUE-ON \\ Mathematics and Applied Mathematics Research Unit, \\ Department of Mathematics, Faculty of Science, Mahasarakham University, \\ Maha Sarakham, 44150, THAILAND
}

\begin{abstract}
In this work, an incorporated form of Sadik transform and Adomian decomposition method which is called the Sadik decomposition method is presented. The method is applied to solve a system of nonlinear fractional Volterra integro-differential equations in the convolution form. To avoid collecting the noise terms that lead the method to fail for seeking the solution, the proposed method is modified by selecting a suitable initial solution. The obtained results are expressed in the explicit form of a power series with easily computable terms. In addition,illustrativeexamplesareshowntodemonstratetheeffectivenessofthemethod.
\end{abstract}

Key-Words: a Nonlinear System, Volterra Integro-Differential Equations, Sadik Transform, Integral transform, Caputofractionalderivative,

Received: January 12, 2021. Revised: June 21, 2021. Accepted: July 10, 2021. Published: August 5, 2021.

\section{Introduction}

Fractional calculus is initially introduced by Leibniz in 1695 [4]. It deals with the derivative and integral of fractional order. The definition of fractional derivatives is given by many mathematicians e.g. RiemannLiouville, Grünwald-Letnikow, Caputo and Generalized Functions Approach [6]. Here we focus on the Caputo idea since it is more useful and clear in the real-life application. One of the most popular uses of fractional derivatives is the modeling of physical problems in the form of fractional differential equations and fractional integro-differential equations.

The fractional integro-differential equation is a generalization of the integro-differential equation of order integer. This kind of equation can be found in various physical applications such as heat conduction in materials with memory. Not only fractional integro-differential equations but also a system of fractional integro-differential equations appear in many mathematical modeling problems of many phenomena. In recent years, the problem of finding a solution to such a system is attracted many researchers. Unfortunately, most systems of fractional integro-differential equations do not have exact analytic solutions. Therefore, an approximate solution for these systems has been investigating by many numerical techniques. To mention a few, Adomian decomposition method (ADM)[ா], Least squares method [], Chebyshev spectral method [3], Generalized Spline Approach [I3], Chebyshev wavelet method [], Chebyshev pseudo-spectral method [ए]] and reference therein.

The Sadik transform was defined by S.L. Shaikh [I6] in 2018. This sort of transformation is a generalization of many integral transforms such as the Laplace Transform, Aboodh Transform, Kamal Transform, Sumudu Transform, Elzaki Transform, and Tarig Transform. The compulsive theorems for Sadik transform were investigated in [피, [피] [ㄱ] ]. The Sadik decomposition method (SDM) is a convenient and powerful mathematical tool in solving various types of problems in science, technology, and engineering. As linearization and discretization are not required in the procedure, this new integral transform method is attractive by many researchers for solving different kinds of problems. Some applications of

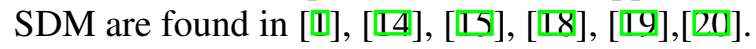

The motivation of this study relates to the progression of work in [ZT]. Besides the theorem of Sadik transform for the fractional derivative is proved, the sufficient conditions for the existence of Sadik transform of fractional derivatives are established. The present paper deals with a system of nonlinear fractional Volterra integro-differential equations (SF- 
VIDEs)

$$
\begin{gathered}
D^{\gamma_{i}} u_{i}(x)=g_{i}(x) \\
+\lambda_{i} \int_{0}^{x} \kappa_{i}(x, t) F_{i}\left(t, u_{1}(t), u_{2}(t), \ldots, u_{n}(t)\right) d t
\end{gathered}
$$

$i=1,2,3, \ldots, n$, where $D^{\gamma_{i}}$ is fractional derivative operator in Caputo sense, $F_{i}$ is a nonlinear function, $g_{i}(x)$ is a given function, $c_{i, 0}, \lambda_{i}$ and $\gamma_{i}$ are constant, $0<\gamma_{i}<1$. This kind of system plays a vital role in many areas of science and engineering [ [] . Some attentions have been paid to the approximate solution of the system [可, [ए]].

The purpose of the paper is to give a solution of the initial value problem for SFVIDE

$$
\begin{gathered}
D^{\gamma_{i}} u_{i}(x)=g_{i}(x) \\
+\lambda_{i} \int_{0}^{x} \kappa_{i}(x-t) F_{i}\left(t, u_{1}(t), u_{2}(t), \ldots, u_{n}(t)\right) d t
\end{gathered}
$$

with initial condition $u_{i}(0)=c_{i, 0}$, by modified Sadik decomposition method. The existence solution of the previous initial value problem is studied in [ [Z3].

This paper is organized as the follows. Section 2 gives the basic definition of fractional integral and derivative whereas section 3 provides the elementary definition, theorem and some properties of the Sadik transform. Section 4 describes how to apply of Sadik decomposition method to SFVIDE. Illustrative examples are shown in the last section.

\section{The Fractional Integral and Derivative}

In this section, some necessary notions about fractional calculus is provided.

Definition 1 The Riemann-Liouville fractional integral operator of order $\gamma \geq 0$ is defined as

$I^{\gamma} \phi(t)=\left\{\begin{array}{l}\frac{1}{\Gamma(\gamma)} \int_{0}^{t} \frac{\phi(\tau)}{(t-\tau)^{1-\gamma}} d \tau, \gamma>0, t>0 \\ \phi(t), \gamma=0\end{array}\right.$

For Riemann-Liouville fractional integral, one can show that

1.) $I^{\gamma_{1}} I^{\gamma_{2}} \phi(t)=I^{\gamma_{1}+\gamma_{2}} \phi(t)$,

2.) $I^{\gamma_{1}} I^{\gamma_{2}} \phi(t)=I^{\gamma_{2}} I^{\gamma_{1}} \phi(t)$,

3.) $I^{\gamma} t^{\mu}=\frac{\Gamma(\mu+1)}{\Gamma(\mu+\gamma+1)} t^{\gamma+\mu}, \quad \mu>-1$.
The Riemann-Liouville fractional integral is a linear operator, that is for any constant $c_{1}, c_{2}$

$$
I^{\gamma}\left(c_{1} \phi(t)+c_{2} \psi(t)\right)=c_{1} I^{\gamma} \phi(t)+c_{2} I^{\gamma} \psi(t) .
$$

Definition 2 [II] The Caputo fractional derivative operator $D^{\gamma}$ of order $\gamma,(n-1<\gamma \leq n, n \in \mathbb{N})$ is defined in the following form,

$$
D^{\gamma} \phi(t)=\frac{1}{\Gamma(n-\gamma)} \int_{0}^{t}(t-\tau)^{-\gamma+n-1} \phi^{(n)}(\tau) d \tau,
$$

$\alpha>0, t>0$, where the function $\phi(t)$ has absolutely continuous derivatives up to order $n-1$. In particular, if $0<\gamma<1$, we have

$$
D^{\gamma} \phi(t)=\frac{1}{\Gamma(n-\gamma)} \int_{0}^{t}(t-\tau)^{-\gamma} \phi^{\prime}(\tau) d \tau .
$$

One can note that Caputo fractional derivative operator is a linear operation

$$
D^{\gamma}\left(c_{1} \phi(t)+c_{2} \psi(t)\right)=c_{1} D^{\gamma} \phi(t)+c_{2} D^{\gamma} \psi(t)
$$

where $c_{1}$ and $c_{2}$ are constants. For the Caputo derivative we have [6]

$D^{\gamma} C=0, C$ is a constant,

$D^{\gamma} x^{p}= \begin{cases}0, & \text { for } p \in \mathbb{N}_{0} \text { and } p<\lceil\gamma\rceil \\ \frac{\Gamma(p+1)}{\Gamma(p+1-\gamma)} x^{p-\gamma}, & \text { for } p \in \mathbb{N}_{0} \text { and } p \geq\lceil\gamma\rceil\end{cases}$

where the ceiling function $\lceil\gamma\rceil$ to denote the smallest integer greater than or equal to $\gamma$ and $\mathbb{N}_{0}=$ $\{0,1,2, \ldots\}$.

Moreover, the following two basic properties can be proved

1.) $D^{\gamma} I^{\gamma} \phi(t)=\phi(t)$,

2.) $I^{\gamma} D^{\gamma} \phi(t)=\phi(t)-\sum_{k=1}^{n-1} \frac{\phi^{(k)}(0)}{k !} t^{k}$.

\section{Sadik Transform}

In this section, we introduce some basic definitions and properties of Sadik transform. As sufficient conditions for the existence of the Sadik transform are that of exponential order, this implies that the Sadik transform may or may not exist. Hence, to guarantee the existence let us consider the set $\mathcal{F}$ defined

$$
\begin{array}{r}
\mathcal{F}=\left\{f(t) \mid \exists M, k_{1}, k_{2}>0 \text { such that }|f(t)| \leq M e^{\frac{|t|}{k_{j}}}\right. \\
\text { if } \left.t \in(-1)^{j} \times[0, \infty), j=1,2\right\}
\end{array}
$$

Then the Sadik transform of exponential ordered function can be stated as follows. 
Definition 3 [16] If $f(t)$ is piecewise continuous function on the interval $0 \leq t \leq A$ for any $A>0$ and $|f(t)| \leq K e^{B t}$ when $t \geq M$, for any real constant $B$ and some positive constant $K$ and $M$. Then Sadik transform of $f(t)$ is defined by

$$
\mathcal{S}[f(t)]=\frac{1}{v^{\beta}} \int_{0}^{\infty} f(t) e^{-t v^{\alpha}} d t=F\left(v^{\alpha}, \beta\right)
$$

where $v$ is complex variable, $\alpha$ is any non zero real number, and $\beta$ is any real number. Here $\mathcal{S}$ is called the Sadik transform operator.

Now the following table shows that the Sadik transform can be convert into the all transforms which are mentioned in Table $\square$ by changing values of $\alpha, \beta$.

Table 1: Sadik transformation with values of $\alpha, \beta$

\begin{tabular}{|c|l|}
\hline Values of $\alpha, \beta$ & Sadik transform converts into \\
\hline$\alpha=1, \beta=0$ & Laplace Transform \\
\hline$\alpha=1, \beta=1$ & Aboodh Transform \\
\hline$\alpha=1, \beta=-1$ & Laplace Carson transform \\
\hline$\alpha=-1, \beta=0$ & Kamal Transform \\
\hline$\alpha=-1, \beta=1$ & Sumudu Transform \\
\hline$\alpha=-1, \beta=-1$ & Elzaki Transform \\
\hline$\alpha=-2, \beta=1$ & Tarig Transform \\
\hline
\end{tabular}

Theorem 4 If $\mathcal{S}[f(t)]=F\left(v^{\alpha}, \beta\right)$ and $\mathcal{S}[g(t)]=$ $G\left(v^{\alpha}, \beta\right)$ then

$$
\mathcal{S}\left[c_{1} f(t)+c_{2} g(t)\right]=c_{1} \mathcal{S}[f(t)]+c_{2} \mathcal{S}[g(t)]
$$

where $c_{1}$ and $c_{2}$ are any constant.

Theorem 5 [10] Let $F\left(v^{\alpha}, \beta\right)$ and $G\left(v^{\alpha}, \beta\right)$ are Sadik Transforms of $f(t)$ and $g(t)$ respectively, and $(f * g)(t)$ is a convolution of $f(t)$ and $g(t)$. Then, Sadik transform of $(f * g)(t)$ is

$$
\mathcal{S}[(f * g)(t)]=v^{\beta} F\left(v^{\alpha}, \beta\right) G\left(v^{\alpha}, \beta\right)
$$

where $*$ denotes convolution.

Theorem 6 [2I] Let $F\left(v^{\alpha}, \beta\right)$ denote the Sadik transform of $f(t)$ and $f^{\prime}(t), f^{\prime \prime}(t), f^{\prime \prime \prime}(t), \ldots$, $f^{(n-1)}(t)$ are continuous on $[0, \infty$.) Then the Sadik transform of $f^{(n)}(t)$ is

$\mathcal{S}\left[f^{(n)}(t)\right]=v^{n \alpha} F\left(v^{\alpha}, \beta\right)-\sum_{k=0}^{n-1} v^{k \alpha-\beta} f^{(n-1-k)}(0)$.
By the above definition and theorem, the following results can be obtained

1. $\mathcal{S}\left[f^{\prime}(t)\right]=v^{\alpha} F\left(v^{\alpha}, \beta\right)-v^{-\beta} f(0)$,

2. $\mathcal{S}\left[f^{\prime \prime}(t)\right]=v^{2 \alpha} F\left(v^{\alpha}, \beta\right)-v^{\alpha-\beta} f(0)-v^{-\beta} f(0)$,

3. $\mathcal{S}\left[f^{\prime \prime \prime}(t)\right]=v^{3 \alpha} F\left(v^{\alpha}, \beta\right)-v^{2 \alpha-\beta} f(0)$

$$
-v^{\alpha-\beta} f^{\prime}(0)-v^{-\beta} f^{\prime \prime}(0),
$$

Moreover, Sadik transform of some functions are shown in Table $\square$. The proof can be found in [16].

Table 2: Sadik transformation of some functions

\begin{tabular}{|c|c|}
\hline$f(t)$ & $\mathcal{S}[f(t)]=F\left(v^{\alpha}, \beta\right)$ \\
\hline 1 & $\frac{1}{v^{\alpha+\beta}}$ \\
\hline$t$ & $\frac{1}{v^{2 \alpha+\beta}}$ \\
\hline$t^{2}$ & $\frac{2 !}{v^{3 \alpha+\beta}}$ \\
\hline$t^{n}, n \in \mathbb{N}$ & $\frac{n !}{v^{(n+1) \alpha+\beta}}$ \\
\hline$t^{n}, n>-1$ & $\frac{\Gamma(n+1)}{v^{(n+1) \alpha+\beta}}$ \\
\hline$e^{a t}$ & $\frac{1}{v^{\beta}\left(v^{\alpha}-a\right)}$ \\
\hline $\sin a t$ & $\frac{a}{v^{\beta}\left(v^{2 \alpha}+a^{2}\right)}$ \\
\hline $\cos a t$ & $\frac{v^{\alpha}}{v^{\beta}\left(v^{2 \alpha}+a^{2}\right)}$ \\
\hline $\sinh a t$ & $\frac{a}{v^{\beta}\left(v^{2 \alpha}-a^{2}\right)}$ \\
\hline $\cosh a t$ & $\frac{v^{\alpha}}{v^{\beta}\left(v^{2 \alpha}-a^{2}\right)}$ \\
\hline
\end{tabular}

Theorem 7 [2I] Let $n-1<\gamma<n,(n=[\gamma]+$ $1)$ and $f(t), f^{\prime}(t), \ldots, f^{(n-1)}(t)$ are continuous on $[0, \infty)$ and of exponential order, while $D^{\gamma} f(t)$ is piecewise continuous on $[0, \infty)$. Then Sadik transform of Caputo fractional derivative of order $\gamma$ of function $f$ is given by

$$
\begin{aligned}
\mathcal{S}\left[D^{\gamma} f(t)\right] & =v^{\gamma \alpha} F\left(v^{\alpha}, \beta\right) \\
& -\sum_{k=0}^{n-1} v^{(\gamma-n+k) \alpha-\beta} f^{(n-1-k)}\left(0^{+}\right)
\end{aligned}
$$




\section{Application of Sadik Decomposi- tion Method to System of Non- linear Fractional Volterra Integro- Differential Equations}

Let us consider the system of nonlinear Volterra integro-differential equations (그) in form

$$
D^{\gamma_{i}} u_{i}(x)=g_{i}(x)+\lambda_{i} \kappa_{i}(x) * F_{i}\left(t, u_{1}, u_{2}, \ldots, u_{n}\right) .
$$

Applying the Sadik transform to (9) and using ([7), one obtains

$$
\begin{array}{r}
\mathcal{S}\left[D^{\gamma_{i}} u_{i}(x)\right]=\mathcal{S}\left[g_{i}(x)\right] \\
+\lambda_{i} v^{\beta} \mathcal{S}\left[\kappa_{i}(x)\right] \mathcal{S}\left[F_{i}\left(x, u_{1}, u_{2}, \ldots, u_{n}\right)\right] .
\end{array}
$$

Let $\mathcal{S}\left[u_{i}(x)\right]=U_{i}\left(v^{\alpha}, \beta\right)$, then by Theorem $\bar{\nabla}$ one gets

$$
\begin{aligned}
U_{i}\left(v^{\alpha}, \beta\right) & =v^{-\gamma_{i} \alpha} \mathcal{S}\left[g_{i}(x)\right]+v^{-\alpha-\beta} c_{i, 0} \\
& +\lambda_{i} v^{-\gamma_{i} \alpha+\beta} \mathcal{S}\left[\kappa_{i}(x)\right] \mathcal{S}\left[F_{i}\left(x, u_{1}, u_{2}, \ldots, u_{n}\right)\right]
\end{aligned}
$$

Taking inverse Sadik transform, then

$$
\begin{array}{r}
u_{i}(x)=\mathcal{S}^{-1}\left[v^{-\gamma_{i} \alpha} \mathcal{S}\left[g_{i}(x)\right]\right]+\mathcal{S}^{-1}\left[v^{-\alpha-\beta} c_{i, 0}\right] \\
+\mathcal{S}^{-1}\left[\lambda_{i} v^{-\gamma_{i} \alpha+\beta} \mathcal{S}\left[\kappa_{i}(x)\right] \mathcal{S}\left[F_{i}\left(x, u_{1}, u_{2}, \ldots, u_{n}\right)\right]\right.
\end{array}
$$

Suppose the solution of (9) expressed in form

$$
u_{i}=\sum_{k=0}^{\infty} u_{i, k}(x)
$$

and nonlinear term $F_{i}\left(x, u_{1}(x), u_{2}(x), \ldots, u_{n}(x)\right)$ is represented by Adomian polynomial $A_{i, k}$

$$
F_{i}\left(x, u_{1}, u_{2}, \ldots, u_{n}\right)=\sum_{k=0}^{\infty} A_{i, k}\left(x, u_{1}, u_{2}, \ldots, u_{n}\right) .
$$

Specific algorithm for multivariables Adomian Polynomials is provided in [8]. Substituting (111) and ([12) into (10), it becomes

$$
\begin{aligned}
\sum_{k=0}^{\infty} u_{i, k}(x) & =\mathcal{S}^{-1}\left[v^{-\gamma_{i} \alpha} \mathcal{S}\left[g_{i}(x)\right]\right]+\mathcal{S}^{-1}\left[v^{-\alpha-\beta} c_{i, 0}\right] \\
& +\mathcal{S}^{-1}\left[\lambda_{i} v^{-\gamma_{i} \alpha+\beta} \mathcal{S}\left[\kappa_{i}(x)\right] \mathcal{S}\left[\sum_{k=0}^{\infty} A_{i, k}\right]\right]
\end{aligned}
$$

Thus, the recursive relation for standard Sadik decomposition method (SSDM) is defined

$$
\begin{aligned}
u_{i, 0}(x) & =\mathcal{S}^{-1}\left[v^{-\gamma_{i} \alpha} \mathcal{S}\left[g_{i}(x)\right]\right]+\mathcal{S}^{-1}\left[v^{-\alpha-\beta} c_{i, 0}\right] \\
& =H_{i}(x)
\end{aligned}
$$

$u_{i, k+1}(x)=\mathcal{S}^{-1}\left[\lambda_{i} v^{-\gamma_{i} \alpha+\beta} \mathcal{S}\left[\kappa_{i}(x)\right] \mathcal{S}\left[A_{i, k}\right]\right]$,

$k=0,1,2, \ldots$, here the function $H_{i}(x)$ represents the term arising from source equation and prescribed initial condition. The initial solution is important, and the choice of $H_{i}(x)$ as the initial solution always leads to noise oscillation during the iteration procedure.

\section{Modified Sadik Decomposition Method (MSDM)}

In order to overcome the shortcoming, we assume that $H_{i}(x)$ can be divided into the sum of two parts namely $H_{i 0}(x)$ and $H_{i 1}(x)$, therefore we get

$$
H_{i}(x)=H_{i 0}(x)+H_{i 1}(x)
$$

Instead of the iteration procedure expressed above one suggests the following modification

$$
\begin{aligned}
& u_{i, 0}(x)=H_{i 0}(x) \text {, } \\
& u_{i, 1}(x)=H_{i 1}(x)+\mathcal{S}^{-1}\left[\lambda_{i} v^{-\gamma_{i} \alpha+\beta} \mathcal{S}\left[\kappa_{i}(x)\right] \mathcal{S}\left[A_{i, 1}\right]\right], \\
& u_{i, k+1}(x)=\mathcal{S}^{-1}\left[\lambda_{i} v^{-\gamma_{i} \alpha+\beta} \mathcal{S}\left[\kappa_{i}(x)\right] \mathcal{S}\left[A_{i, k}\right]\right],
\end{aligned}
$$

\section{Illustrative Examples}

This section gives examples which illustrate an application of MSDM to the system of nonlinear fractional Volterra integro-differential equations.

Example 8 Consider a system of nonlinear fractional Volterra integro-differential equations

$$
\begin{aligned}
& D^{\frac{1}{2}} u(x)=\frac{2 x^{\frac{1}{2}}}{\Gamma\left(\frac{3}{2}\right)}+\frac{x^{4}}{3}+\frac{x^{8}}{56}-\int_{0}^{x}(x-t)\left(u^{2}(t)+w^{2}(t)\right) d t, \\
& D^{\frac{1}{2}} w(x)=\frac{6 x^{\frac{5}{2}}}{\Gamma\left(\frac{7}{2}\right)}+\frac{x^{6}}{15}-\int_{0}^{x}(x-t)(u(t) w(t)) d t
\end{aligned}
$$

with initial conditions

$$
u(0)=0, w(0)=0 .
$$

The exact solution is $u(x)=2 x, w(x)=x^{3}$. 
Here $g_{1}(x)=\frac{2 x^{\frac{1}{2}}}{\Gamma\left(\frac{3}{2}\right)}+\frac{x^{4}}{3}+\frac{x^{8}}{56}, g_{2}(x)=\frac{6 x^{\frac{5}{2}}}{\Gamma\left(\frac{7}{2}\right)}+$ $\frac{x^{6}}{15}, F_{1}(u, w)=u^{2}+w^{2}, F_{2}(u, w)=u w, \gamma_{i}=$ $\frac{1}{2}, \lambda_{i}=-1$ and $\kappa_{i}(x)=x, i=1,2$.

Suppose the solution of this system is written as follows

$$
u=\sum_{n=0}^{\infty} u_{n}(x) \text { and } w=\sum_{n=0}^{\infty} w_{n}(x)
$$

By utilizing SSMD, one can define recursive relation as

$$
\begin{aligned}
u_{0}(x) & =2 x+\frac{8 x^{\frac{9}{2}}}{\Gamma\left(\frac{11}{2}\right)}+\frac{720 x^{\frac{17}{2}}}{\Gamma\left(\frac{19}{2}\right)}, \\
u_{n+1}(x) & =-S^{-1}\left[\frac{1}{v^{\frac{5}{2}} \alpha} \mathcal{S}\left[A_{n}(x)\right], n \geq 0,\right.
\end{aligned}
$$

and

$$
\begin{aligned}
w_{0}(x) & =x^{3}+\frac{48 x^{\frac{13}{2}}}{\Gamma\left(\frac{15}{2}\right)}, \\
w_{n+1}(x) & =-\mathcal{S}^{-1}\left[\frac{1}{v^{\frac{5}{2} \alpha}} \mathcal{S}\left[B_{n}(x)\right], n \geq 0\right.
\end{aligned}
$$

where $A_{n}$ and $B_{n}$ are the Adomian polynomials for nonlinear functions $F_{1}(u, w)$ and $F_{2}(u, w)$ respectively. The specific algorithm for multivariable Adomian polynomials gives [8],

$$
\begin{aligned}
& A_{0}=u_{0}^{2}+w_{0}^{2}, \\
& A_{1}=2 u_{0} u_{1}+2 w_{0} w_{1}, \\
& A_{2}=2 u_{0} u_{2}+2 w_{0} w_{2}+u_{1}^{2}+w_{1}^{2},
\end{aligned}
$$

and

$$
\begin{aligned}
& B_{0}=u_{0} w_{0}, \\
& B_{1}=u_{0} w_{1}+w_{0} u_{1}, \\
& B_{2}=u_{2} w_{0}+w_{2} u_{0}+u_{1} w_{1} .
\end{aligned}
$$

For $n=0$, we have

$$
\begin{aligned}
& u_{1}(x)=-\frac{8 x^{\frac{9}{2}}}{\Gamma\left(\frac{11}{2}\right)}-\frac{11 x^{8}}{2520}-\frac{720 x^{\frac{17}{2}}}{\Gamma\left(\frac{19}{2}\right)}-\frac{(64)(9 !) x^{\frac{23}{2}}}{\Gamma^{2}\left(\frac{11}{2}\right) \Gamma\left(\frac{25}{2}\right)}+\ldots \\
& w_{1}(x)=-\frac{48 x^{\frac{13}{2}}}{\Gamma\left(\frac{15}{2}\right)}-\frac{19 x^{10}}{43200}-\frac{(192)(11 !) x^{\frac{27}{2}}}{\Gamma\left(\frac{11}{2}\right) \Gamma\left(\frac{15}{2}\right) \Gamma\left(\frac{29}{2}\right)}+\ldots
\end{aligned}
$$

We can note that the noise terms appeared and these will lead to the complexity computation in the next iteration. On the other side, by applying MSDM, the recursive relation is defined by

$$
\begin{aligned}
u_{0}(x) & =2 x, \\
u_{1}(x) & =\frac{8 x^{\frac{9}{2}}}{\Gamma\left(\frac{11}{2}\right)}+\frac{720 x^{\frac{17}{2}}}{\Gamma\left(\frac{19}{2}\right)}-\mathcal{S}^{-1}\left[\frac{1}{v^{\frac{5}{2}} \alpha} \mathcal{S}\left[A_{0}(x)\right]\right], \\
u_{n+1}(x) & =-S^{-1}\left[\frac{1}{v^{\frac{5}{2} \alpha}} \mathcal{S}\left[A_{n}(x)\right]\right], n \geq 1,
\end{aligned}
$$

and

$$
\begin{aligned}
w_{0}(x) & =x^{3}, \\
w_{1}(x) & =\frac{48 x^{\frac{13}{2}}}{\Gamma\left(\frac{15}{2}\right)}-\mathcal{S}^{-1}\left[\frac{1}{v^{\frac{5}{2} \alpha}} \mathcal{S}\left[B_{0}(x)\right]\right], \\
w_{n+1}(x) & =-\mathcal{S}^{-1}\left[\frac{1}{v^{\frac{5}{2} \alpha}} \mathcal{S}\left[B_{n}(x)\right]\right], n \geq 1
\end{aligned}
$$

By direct calculation, the components of the unknown functions $u_{n}(x)$ and $w_{n}(x)$ are given

$$
\begin{array}{r}
u_{0}(x)=2 x, w_{0}(x)=x^{3} \\
u_{n}(x)=0, w_{n}(x)=0, n \geq 1 .
\end{array}
$$

By ([13), the solution of this problem is $u(x)=$ $2 x, w(x)=x^{3}$.

Example 9 Now, consider the system of nonlinear fractional Volterra integro-differential equations

$$
\begin{aligned}
& D^{\frac{1}{2}} u(x)=\frac{x^{\frac{1}{2}}}{\Gamma\left(\frac{3}{2}\right)}+\frac{x^{4}}{12}+\frac{2 x^{6}}{15}-\int_{0}^{x}(x-t)\left(u^{2}(t)+w^{2}(t)\right) d t \\
& D^{\frac{2}{3}} w(x)=\frac{4 x^{\frac{4}{3}}}{\Gamma\left(\frac{7}{3}\right)}+\frac{2 x^{6}}{15}-\frac{x^{4}}{12}-\int_{0}^{x}(x-t)\left(w^{2}(t)-u^{2}(t)\right) d t
\end{aligned}
$$

\section{subject to the initial conditions}

$$
u(0)=0, w(0)=0,
$$

which exact solution is $u(x)=x, w(x)=2 x^{2}$.

Note that $g_{1}(x)=\frac{x^{\frac{1}{2}}}{\Gamma\left(\frac{3}{2}\right)}+\frac{x^{4}}{12}+\frac{2 x^{6}}{15}, g_{2}(x)=\frac{4 x^{\frac{4}{3}}}{\Gamma\left(\frac{7}{3}\right)}+$ $\frac{2 x^{6}}{15}-\frac{x^{4}}{12}, F_{1}(u, w)=u^{2}+w^{2}, F_{2}(u, w)=w^{2}-$ $u^{2}, \gamma_{1}=\frac{1}{2}, \gamma_{2}=\frac{2}{3}, \lambda_{1}=\lambda_{2}=-1$ and $\kappa_{i}(x)=$ $x$. Suppose the solution of this system is written as follows

$$
u=\sum_{n=0}^{\infty} u_{n}(x) \text { and } \quad w=\sum_{n=0}^{\infty} w_{n}(x)
$$


After applying Standard Sadik decomposition method, the recursive relation is defined by

$$
\begin{aligned}
u_{0}(x) & =x+\frac{2 x^{\frac{9}{2}}}{\Gamma\left(\frac{11}{2}\right)}+\frac{96 x^{\frac{13}{2}}}{\Gamma\left(\frac{15}{2}\right)}, \\
u_{n+1}(x) & =-\mathcal{S}^{-1}\left[\frac{1}{v^{\frac{5}{2} \alpha}} \mathcal{S}\left[A_{n}(x)\right]\right], n \geq 0
\end{aligned}
$$

and

$$
\begin{gathered}
w_{0}(x)=2 x^{2}+\frac{96 x^{\frac{20}{3}}}{\Gamma\left(\frac{23}{3}\right)}-\frac{2 x^{\frac{14}{3}}}{\Gamma\left(\frac{17}{3}\right)}, \\
w_{n+1}(x)=-\mathcal{S}^{-1}\left[\frac{1}{v^{\frac{8}{3} \alpha}} \mathcal{S}\left[B_{n}(x)\right]\right], n \geq 0
\end{gathered}
$$

where $A_{n}$ and $B_{n}$ are the Adomian polynomials for nonlinear functions $F_{1}(u, w)$ and $F_{2}(u, w)$ respectively. Here,

$$
\begin{aligned}
& A_{0}=u_{0}^{2}+w_{0}^{2}, \\
& A_{1}=2 u_{0} u_{1}+2 w_{0} w_{1}, \\
& A_{2}=2 u_{0} u_{2}+2 w_{0} w_{2}+u_{1}^{2}+w_{1}^{2},
\end{aligned}
$$

and

$$
\begin{aligned}
& B_{0}=w_{0}^{2}-u_{0}^{2}, \\
& B_{1}=2 w_{0} w_{1}-2 u_{0} u_{1}, \\
& B_{2}=2 w_{0} w_{2}-2 u_{0} u_{2}+w_{1}^{2}-u_{1}^{2},
\end{aligned}
$$

After one step, we obtain

$$
\begin{aligned}
& u_{1}(x)=-\frac{2 x^{\frac{9}{2}}}{\Gamma\left(\frac{11}{2}\right)}-\frac{96 x^{\frac{13}{2}}}{\Gamma\left(\frac{15}{2}\right)}-\frac{11 x^{8}}{20160}+\frac{2720 x^{\frac{55}{6}}}{9 \Gamma\left(\frac{61}{6}\right)}+\ldots, \\
& w_{1}(x)=\frac{2 x^{\frac{14}{3}}}{\Gamma\left(\frac{17}{3}\right)}-\frac{96 x^{\frac{20}{3}}}{\Gamma\left(\frac{23}{3}\right)}+\frac{22 x^{\frac{49}{6}}}{\Gamma\left(\frac{55}{6}\right)}+\frac{2720 x^{\frac{28}{3}}}{9 \Gamma\left(\frac{31}{3}\right)}+\ldots
\end{aligned}
$$

The noise terms are found. Therefore, we apply the MSDM, the recursive relation is defined by

$$
\begin{aligned}
u_{0}(x) & =\mathcal{S}^{-1}\left[\frac{1}{v^{2 \alpha+\beta}}\right]=x, \\
u_{1}(x) & =\frac{2 x^{\frac{9}{2}}}{\Gamma\left(\frac{11}{2}\right)}+\frac{96 x^{\frac{13}{2}}}{\Gamma\left(\frac{15}{2}\right)}-\mathcal{S}^{-1}\left[\frac{1}{v^{\frac{5}{2} \alpha}} \mathcal{S}\left[A_{0}(x)\right]\right], \\
u_{n+1}(x) & =-\mathcal{S}^{-1}\left[\frac{1}{v^{\frac{5}{2} \alpha}} \mathcal{S}\left[A_{n}(x)\right]\right],
\end{aligned}
$$

and

$$
\begin{aligned}
w_{0}(x) & =\mathcal{S}^{-1}\left[\frac{4}{v^{3 \alpha+\beta}}\right]=2 x^{2}, \\
w_{1}(x) & =\frac{96 x^{\frac{20}{3}}}{\Gamma\left(\frac{23}{3}\right)}-\frac{2 x^{\frac{14}{3}}}{\Gamma\left(\frac{17}{3}\right)}-\mathcal{S}^{-1}\left[\frac{1}{v^{\frac{8}{3} \alpha}} \mathcal{S}\left[B_{0}(x)\right]\right], \\
w_{n+1}(x) & =-\mathcal{S}^{-1}\left[\frac{1}{v^{\frac{8}{3} \alpha}} \mathcal{S}\left[B_{n}(x)\right]\right], n \geq 1 .
\end{aligned}
$$

By direct calculation, the components of the unknown functions $u_{n}(x)$ and $w_{n}(x)$ are given

$$
\begin{array}{r}
u_{0}(x)=x, w_{0}(x)=2 x^{2}, \\
u_{n}(x)=0, w_{n}(x)=0, n \geq 1 .
\end{array}
$$

By (I4), the solution of this problem is $u(x)=x$, $w(x)=2 x^{2}$.

Example 10 Consider the following system of nonlinear fractional Volterra integro-differential equations

$$
\begin{aligned}
D^{\gamma_{1}} u(x) & =\frac{x^{1-\gamma_{1}}}{\Gamma\left(2-\gamma_{1}\right)}-\frac{2 x^{2-\gamma_{1}}}{\Gamma\left(3-\gamma_{1}\right)}-4 x^{2} \\
& +\int_{0}^{x}(x-t)(u(t)+w(t))^{3} d t, \\
D^{\gamma_{2}} w(x) & =\frac{2 x^{2-\gamma_{2}}}{\Gamma\left(3-\gamma_{2}\right)}-\frac{x^{1-\gamma_{2}}}{\Gamma\left(2-\gamma_{2}\right)}-x^{4} \\
& +\int_{0}^{x}(x-t)^{3}\left(u^{2}(t)+2 u(t) w(t)+w^{2}(t)\right) d t
\end{aligned}
$$

subject to

$$
u(0)=1, w(0)=1,
$$

where $0<\gamma_{1}, \gamma_{2} \leq 1$. The exact solution of this system is $u(x)=1+x-x^{2}, w(x)=1-x+x^{2}$.

Here, we have $g_{1}(x)=\frac{x^{1-\gamma_{1}}}{\Gamma\left(2-\gamma_{1}\right)}-\frac{2 x^{2-\gamma_{1}}}{\Gamma\left(3-\gamma_{1}\right)}-4 x^{2}$, $g_{2}(x)=\frac{2 x^{2-\gamma_{2}}}{\Gamma\left(3-\gamma_{2}\right)}-\frac{x^{1-\gamma_{2}}}{\Gamma\left(2-\gamma_{2}\right)}-x^{4}, \kappa_{1}(x)=x, \kappa_{2}(x)=$ $x^{3}, F_{1}(u, w)=(u+w)^{3}$ and $F_{2}(u, w)=(u+w)^{2}$. Let the solution of this problem be represented by

$$
u=\sum_{n=0}^{\infty} u_{n}(x) \quad \text { and } \quad w=\sum_{n=0}^{\infty} w_{n}(x)
$$

By using SSDM, the recursive relation for this problem are

$$
\begin{aligned}
u_{0}(x) & =1+x-x^{2}-\frac{8 x^{2+\gamma_{1}}}{\Gamma\left(3+\gamma_{1}\right)}, \\
u_{n+1}(x) & =\mathcal{S}^{-1}\left[\frac{1}{v^{\left(2+\gamma_{1}\right) \alpha}} \mathcal{S}\left[A_{n}(x)\right]\right], n \geq 0
\end{aligned}
$$

and

$$
\begin{aligned}
w_{0}(x) & =1-x+x^{2}-\frac{24 x^{4+\gamma_{2}}}{\Gamma\left(5+\gamma_{2}\right)}, \\
w_{n+1}(x) & =\mathcal{S}^{-1}\left[\frac{6}{v^{\left(4+\gamma_{2}\right) \alpha}} \mathcal{S}\left[B_{n}(x)\right]\right], n \geq 0
\end{aligned}
$$


where $A_{n}$ and $B_{n}$ are the Adomian polynomials for nonlinear functions $F_{1}(u, w)$ and $F_{2}(u, w)$ respectively. Here,

$$
\begin{aligned}
A_{0}= & \left(u_{0}+w_{0}\right)^{3}, \\
A_{1}= & 3\left(u_{1}+w_{1}\right)\left(u_{0}+w_{0}\right)^{2}, \\
A_{2}= & 3\left(u_{2}+w_{2}\right)\left(u_{0}+w_{0}\right)^{2}+3\left(u_{0}+w_{0}\right)\left(u_{1}^{2}+w_{1}^{2}\right) \\
& +6 u_{1} w_{1}\left(u_{0}+w_{0}\right),
\end{aligned}
$$

and

$$
\begin{aligned}
& B_{0}=\left(u_{0}+w_{0}\right)^{2}, \\
& B_{1}=2\left(u_{1}+w_{1}\right)\left(u_{0}+w_{0}\right), \\
& B_{2}=2\left(u_{2}+w_{2}\right)\left(u_{0}+w_{0}\right)+u_{1}^{2}+w_{1}^{2}+2 u_{1} w_{1} .
\end{aligned}
$$

After one iteration, we get

$$
\begin{aligned}
u_{1}(x) & =\frac{8 x^{2+\gamma_{1}}}{\Gamma\left(3+\gamma_{1}\right)}-\frac{96 x^{2\left(2+\gamma_{1}\right)}}{\Gamma\left(5+2 \gamma_{1}\right)}+\ldots, \\
w_{1}(x) & =\frac{24 x^{4+\gamma_{2}}}{\Gamma\left(5+\gamma_{2}\right)}-\frac{192 x^{6+\gamma_{1}+\gamma_{2}}}{\Gamma\left(7+\gamma_{1}+\gamma_{2}\right)}+\ldots
\end{aligned}
$$

Because the noise terms are detected, the MSDM is considerate and the recursive relation are

$$
\begin{aligned}
u_{0}(x) & =1+x \\
u_{1}(x) & =-x^{2}-\frac{8 x^{2+\gamma_{1}}}{\Gamma\left(3+\gamma_{1}\right)}+\mathcal{S}^{-1}\left[\frac{1}{v^{\left(2+\gamma_{1}\right) \alpha}} \mathcal{S}\left[A_{0}(x)\right]\right], \\
u_{n+1}(x) & =\mathcal{S}^{-1}\left[\frac{1}{v^{\left(2+\gamma_{1}\right) \alpha}} \mathcal{S}\left[A_{n}(x)\right]\right]
\end{aligned}
$$

and

$$
\begin{aligned}
w_{0}(x) & =1-x \\
w_{1}(x) & =x^{2}-\frac{24 x^{4+\gamma_{2}}}{\Gamma\left(5+\gamma_{2}\right)}+\mathcal{S}^{-1}\left[\frac{6}{v^{\left(4+\gamma_{2}\right) \alpha}} \mathcal{S}\left[B_{0}(x)\right]\right], \\
w_{n+1}(x) & =\mathcal{S}^{-1}\left[\frac{6}{v^{\left(4+\gamma_{2}\right) \alpha}} \mathcal{S}\left[B_{n}(x)\right]\right], n \geq 1 .
\end{aligned}
$$

Using the above relations, we easily obtain the components of the unknown functions $u_{n}(x)$ and $w_{n}(x)$,

$$
\begin{aligned}
& u_{0}(x)=1+x, w_{0}(x)=1-x, \\
& u_{1}(x)=-x^{2}, w_{1}(x)=x^{2} \\
& u_{n}(x)=0, w_{n}(x)=0, n \geq 2 .
\end{aligned}
$$

By (ㅍ)), we found that the solution of this problem is $u(x)=1+x-x^{2}, w(x)=1-x+x^{2}$.

\section{Discussion}

The initial approximate solution in the iteration is crucial for applying the SDM to the SFVIDEs. As seen in the previous examples, the iteration for SSDM always constructs the noise terms from the first iteration. Gradually accumulated noise terms in each iterative step can be caused to the approximate series solution to converge slowly or even diverge. However, these concerns can be managed in the MSDM iteration by selecting the suitable initial approximation. In the MSDM iteration, the noise oscillating terms are eliminated and the solution can be found in a few iteration steps with easily computable terms. The key to choosing the proper initial approximate solution is trying a simple part of non-homogeneous terms that satisfied the initial conditions.

\section{Conclusion}

This work shows that we have succeeded in searching for the solution of the SFVIDEs by the MSDM. The advantage of the MSDM is constructing a productive approximate series solution in a few iterations. The MSDM is also practical and convenient to use. The effectiveness of the method is performed by the initial problem for the SFVIDEs involving a convolution form. Others form of SFVIDES has been investigating.

Acknowledgements: This research project was financially supported by Mahasarakham University 2021. The author is deeply indebted to all reviewers for their valuable comments and suggestions. Thanks to Ms. Nutsuda Chaiseeha and Ms. Wannaporn Kamonpob for their help.

\section{References:}

[1] A. Sudhanshu, R.G. Anjana and D.S. Swarg, Application of Sadik Transform for Handling Linear Volterra Integro-Differential Equations of Second Kind, Universal Review, Vol.10, No.7, 2019, pp.177-187.

[2] A.M.S. Mahdy, Numerical studies for solving fractional integro-differential equations, Journal of Ocean Engineering and Science, Vol.3, 2018, 127-132.

[3] A.Z. Hassan, Sh.T. Seham and M.S. Yara, New solutions for system of fractional integrodifferential equations and Abels integral equations by Chebyshev spectral method, Mathematical Problems in Engineering, vol. 2017, Article ID 7853839.

[4] F. Awawdeh, E.A. Rawashdeh and H.M. Jaradat, Analytic solution of fractional integrodifferential equations, Annals of the University 
of Craiova, Mathematics and Computer Science Series, Vol.38, No.1, 2011, pp. 1-10.

[5] H. Khan, M. Arif, S.T. Mohyud-Din and S. Bushnaq, Numerical solutions to systems of fractional Voltera Integro differential equations, using Chebyshev wavelet method, Journal of Taibah University for Science, Vol.12, No.5, 2018, pp. 584-591.

[6] I. Podlubny, Fractional differential equations: an introduction to fractional derivatives, fractional differential equations, to methods of their solution and some of their applications, New York: Academic Press, 1999.

[7] J.R. Loh and C. Phang, A new numerical scheme for solving system of Volterra integrodifferential equation, Alexandria Engineering Journal, Vol.57, No.2, 2018, pp. 1117-1124.

[8] K. Abbaoui, Y. Cherruault and V. Seng, Practical formulae for the calculating of multivariable Adomian polynomials, Math. Comput. Modelling, Vol.22, 1995, pp. 89-93.

[9] K. Parand and M. Delkhosh, System of Nonlinear Volterra Integro-Differential Equations of Arbitrary Order, Boletim da Sociedade Paranaense de Matematica, Vol.36, No.4, 2018, pp.33-54.

[10] M.H. Saleh, D.Sh. Mohamed, M.H. Ahmed and M.K. Marjan, System of linear fractional integro-differential equations by using Adomian decomposition method, International Journal of Computer Applications, Vol.121, No.24, 2015, pp. 9-19.

[11] M.M. Khader and N.H. Sweilam, On the approximate solutions for system of fractional integrodifferential equations using Chebyshev pseudospectral method, Applied Mathematical Modelling, Vol.37, No.24, 2013, pp. 9819-9828.

[12] M. Zurigat, Sh. Momani and Ah. Alawneh, Homotopy analysis method for system of fractional integro-differential equations, Neural, Parallel, and Scientific Computations, Vol.17, 2009, pp. 169-186.

[13] N.N. Hasan and D.A. Hussien, Generalized Spline Approach for Solving System of Linear Fractional Volterra Integro-Differential Equations, Ibn Al Haitham Journal for Pure and Applied sciences., Vol.31, No.1, 2018, pp.222-230.
[14] S. Aggarwal and K. Bhatnagar, Sadik Transform for Handling Population Growth and Decay Problems, Journal of Applied Science and Computations, Vol.6, No.6, 2019, pp. 12121221.

[15] S.K. Ganesh and S.A. Nitin, Solution to linear partial integro-differential equation by using Sadik Transform, Journal of Applied Science and Computations, Vol.6, No.4, 2019, pp.639645.

[16] S.L. Shaikh, Introducing a new integral transform: Sadik Transform, American International Journal of Research in Science, Technology, Engineering \& Mathematics, Vol.22, No.1, 2018, pp. 100-102.

[17] S.L. Shaikh, Sadik transform in control theory, International Journal of Innovative Science and Research Technology, Vol.3, No.5, 2018, pp.396-398.

[18] S.L. Shaikh, Some applications of the new integral transform for partial differential equations, Mathematical Journal of Interdisciplinary Sciences, Vol.7, No.1, 2018, pp. 45-49.

[19] S.P. Shivaji and S.A. Nitin, Applications of Sadik Transform for solving Bessels function and linear Volterra integral equation of convolution type, Multidisciplinary Research, Vol.6, No.3, 2019, pp.85-91.

[20] S.S. Redhwan, S.L. Shaikh and M.S. Abdo, On a study of some new results in fractional calculus through Sadik transform, National Conference on Recent Trends in Physics, Chemistry and Mathematics (RTPCM-2020), Vol.68, No.38, 2020, pp.681-693.

[21] S.S. Redhwan, S.L. Shaikh and M.S. Abdo, Some properties of Sadik transform and its applications of fractional-order dynamical systems in control theory, Advances in the Theory of Nonlinear Analysis and its Applications, Vol.4, No.1, 2020, pp.51-66.

[22] Y. Singh, Theorems and Properties of Sadik Transform, Multidisciplinary, Vol.4, No.5, 2019, pp.1541-1545.

[23] Z. Dahmani, M.A. Abdellaoui and M. Houas, Coupled Systems of Fractional IntegroDifferential Equations Involving Several Functions, Theory and Applications of Mathematics \& Computer Science, Vol.5, No.1, 2015, pp. 53-61. 


\section{Sources of funding for research pre- sented in a scientific article or scientific article itself}

This research project was financially supported by Mahasarakham University 2021.

Creative Commons Attribution License 4.0 (Attribution 4.0 International , CC BY 4.0)

This article is published under the terms of the Creative Commons Attribution License 4.0

https://creativecommons.org/licenses/by/4.0/deed.en_US 